Supporting Information

\title{
Activating Three-dimensional Networks of Fe@Ni Nanofibers via Fast Surface Modification for Efficient Overall Water Splitting
}

Jingying Tao ${ }^{1,2}$, Yijie Zhang ${ }^{1}$, Shengping Wang ${ }^{1}$, Ge Wang ${ }^{1}$, Fei $\mathrm{Hu}^{1}$, Xiaojun Yan ${ }^{1}$, Lifeng $\mathrm{Hao}^{2, *}$, Zhijun Zuo ${ }^{3, *}$, Xiaowei Yang ${ }^{1,4^{*}}$

1. School of Materials Science and Engineering, Tongji University, Shanghai 200123, China

2. Department of Engineering, Qiuzhen School, Huzhou University, Huzhou 313000, China

3. Key Laboratory of Coal Science and Technology of Ministry of Education and Shanxi Province, Taiyuan University of Technology, Taiyuan 030024, Shanxi, China

4. School of Materials Science and Engineering, Chang'an University, Xi'an 710064, China

${ }^{*}$ Corresponding authors. E-mail: yangxw@tongji.edu.cn (X. Yang); 02578@zjhu.edu.cn (L. Hao); zuozhijun@tyut.edu.cn (Z.Zuo)

KEYWORDS: Three-dimensional network; NiFe nanofiber; fast surface modification; self-supporting electrode; overall water splitting 
a)

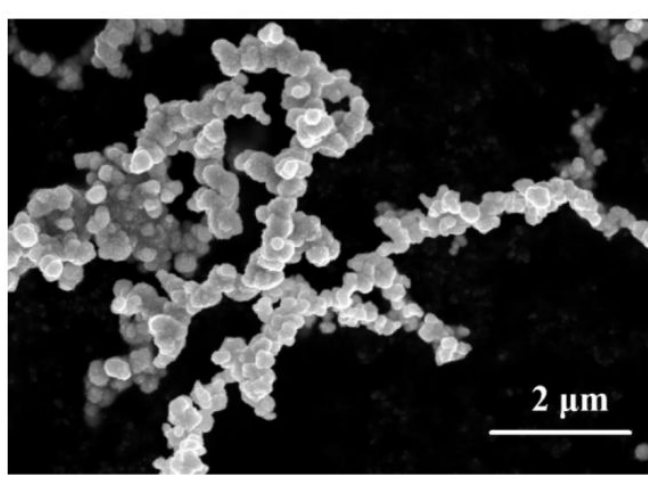

b)

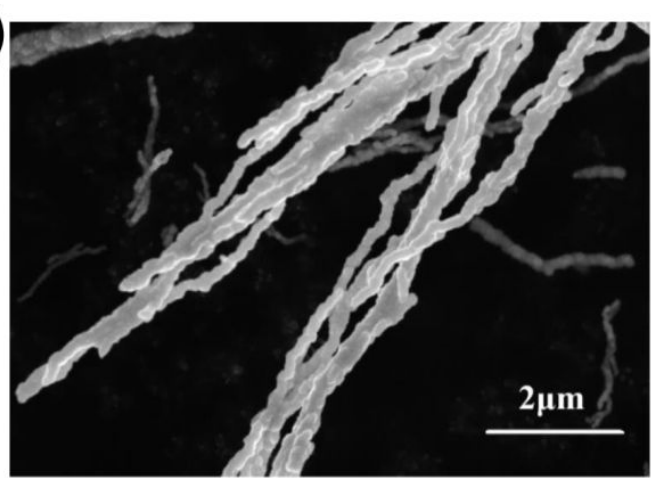

Figure S1. Scanning electron microscopy (SEM) images of Ni nanofiber electrode prepared (a) without and (b) with external magnetic field. The material prepared without parallel magnetic field presents a relatively larger size in diameter comparing with random distribution of these Ni particles. While for the one prepared with the aid of external magnetic field, the resultant materials are aligned along the magnetic field, showing obvious nanofiber morphology.

a)

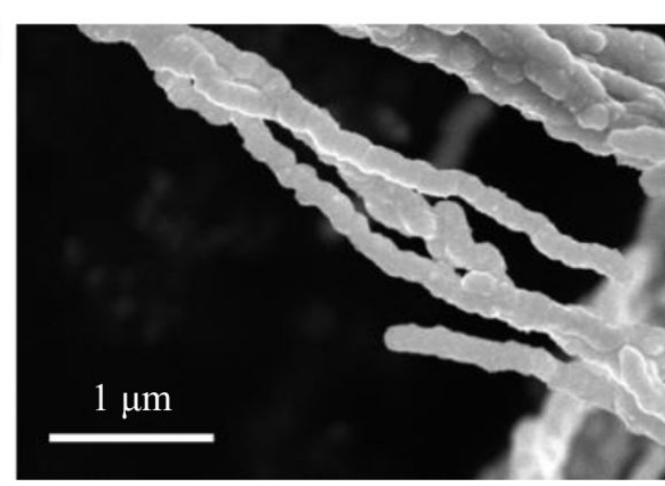

b)

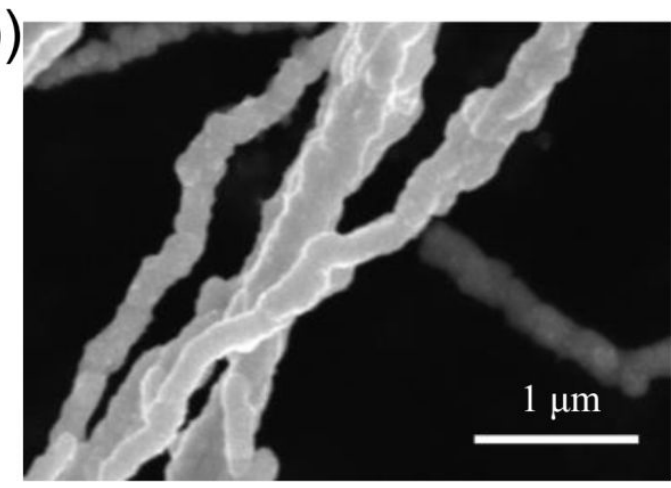

Figure S2. Scanning electron microscopy (SEM) images of (a) Fe@Ni nanofiber electrode (b) Ni nanofiber electrode. Negligible change in morphology is shown after the surface modification process. 

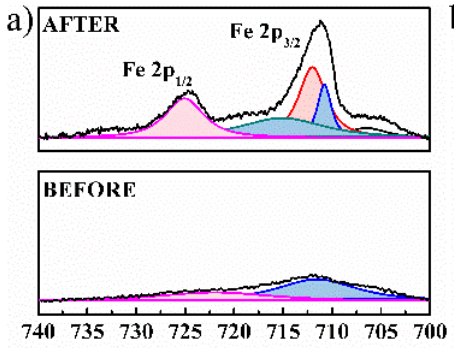

Binding Energy (cV)
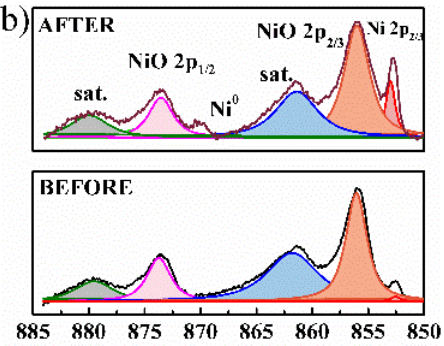

Binding energy $(\mathrm{cV})$
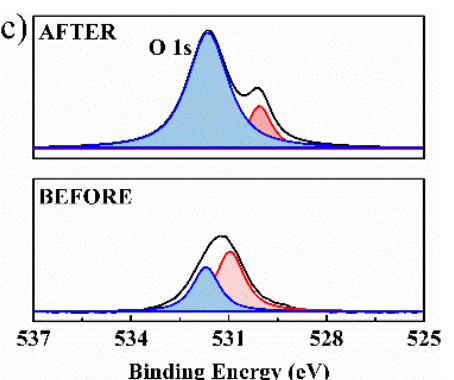

Figure S3. XPS high-resolution scans of Ni nanofiber electrode before and after surface modification: a) Fe, b) Ni, c) O.
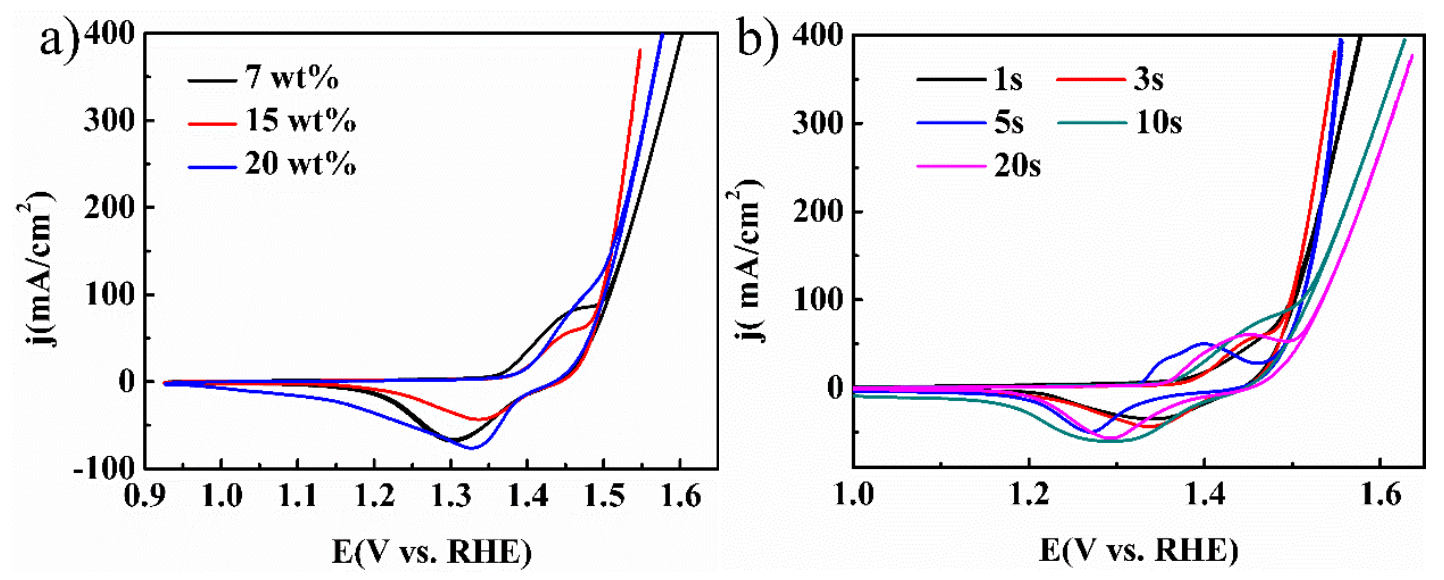

Figure S4. The CV plots of Fe@Ni nanofiber networks obtained under (a) different concentration of $\mathrm{FeCl}_{3}$ for 3 seconds; (b) different etching time in $15 \mathrm{wt} \% \mathrm{FeCl}_{3}$. All of the $\mathrm{CV}$ tests are conducted with a scan rate of $5 \mathrm{mV} / \mathrm{s}$. The results show that the OER performances vary under different $\mathrm{FeCl}_{3}$ concentration as well as etching time and the sample treated by $15 \mathrm{wt} \% \mathrm{FeCl}_{3}$ for 3 seconds is chosen for its optimal OER performance. 

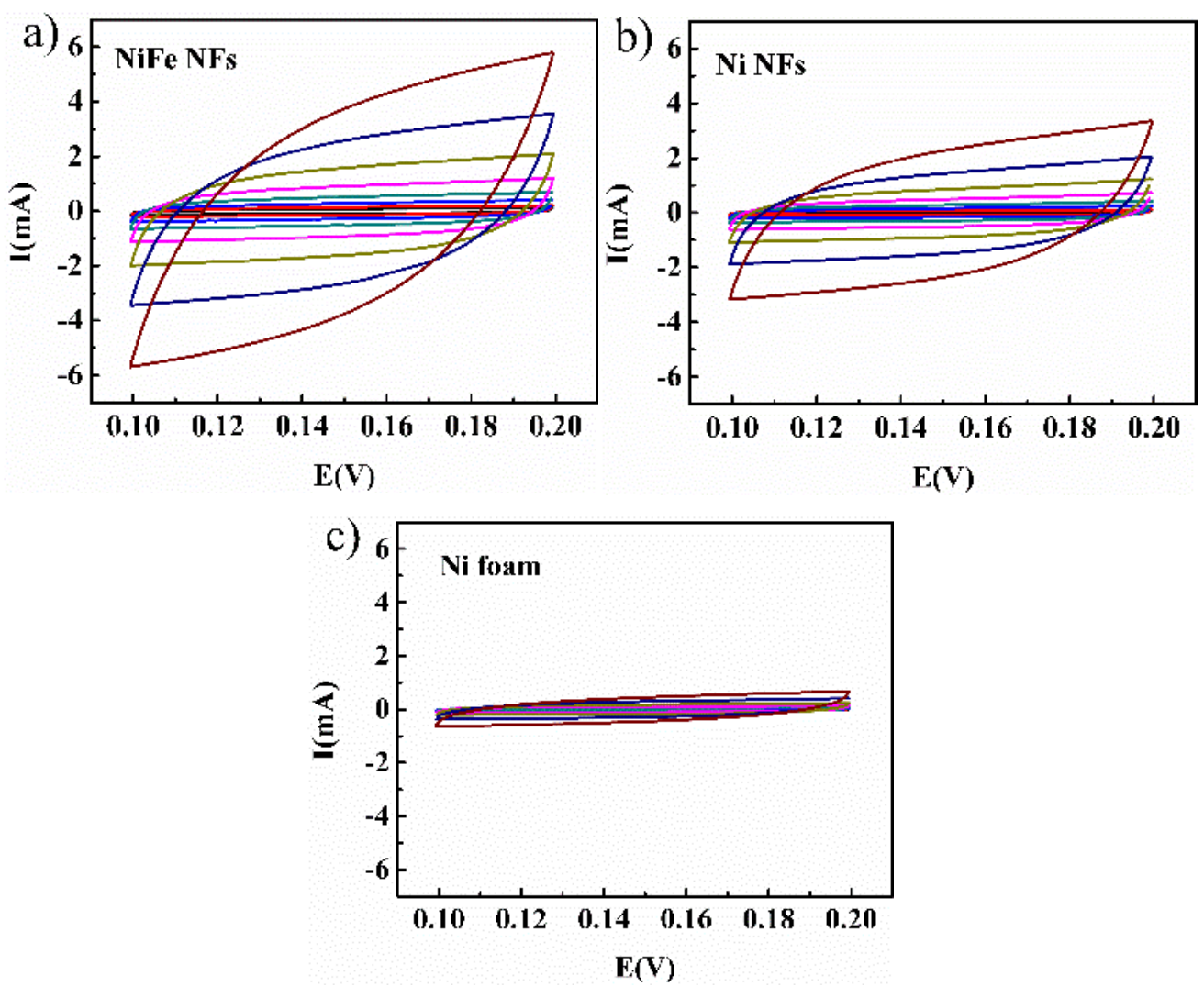

Figure S5. CV curves of different free-standing electrodes. (a) Fe@Ni nanofiber electrode, (b)Ni nanofiber electrode, (c)Ni foam tested in the region of 0.1-0.2 V vs. $\mathrm{Hg} / \mathrm{HgO}$. The scan rates are 5, 10, 25, 50, 100, 200, 400, $800 \mathrm{mV} \mathrm{s}^{-1}$.
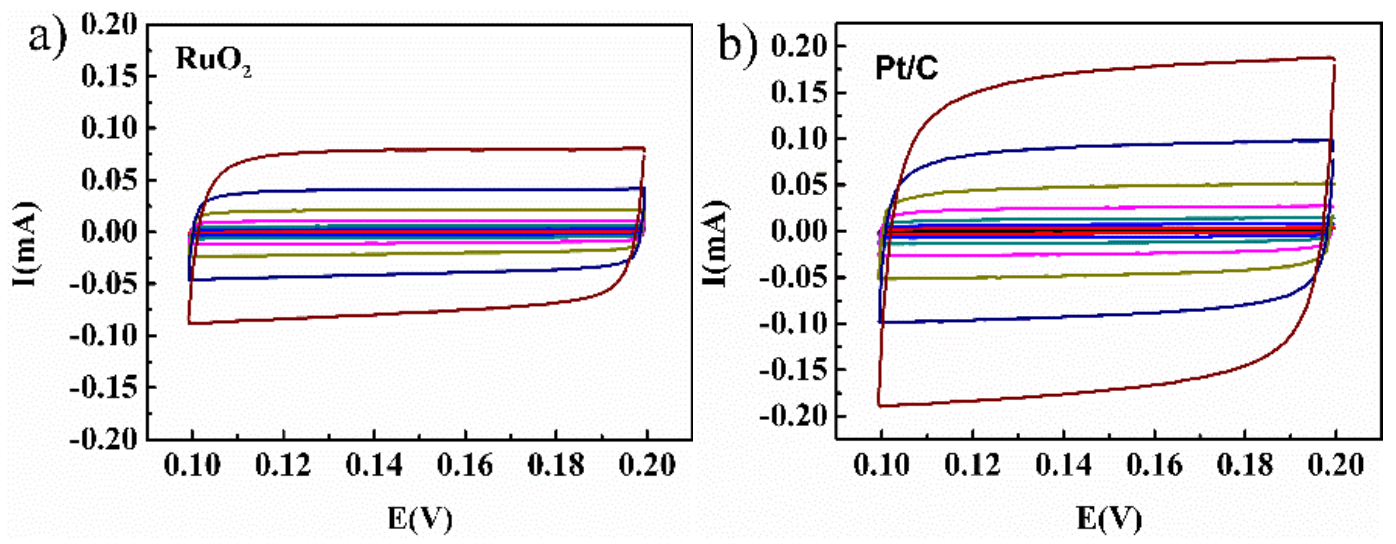

Figure S6. Cyclic voltamogram (CV) curves of benchmark precious-metal electrocatalysts. (a) $\mathrm{RuO}_{2}$, (b) $\mathrm{Pt} / \mathrm{C}$ tested in the region of $0.1-0.2 \mathrm{~V}$ vs. $\mathrm{Hg} / \mathrm{HgO}$. The scan rates are $5,10,25,50,100,200,400,800 \mathrm{mV} \mathrm{s}^{-1}$. 

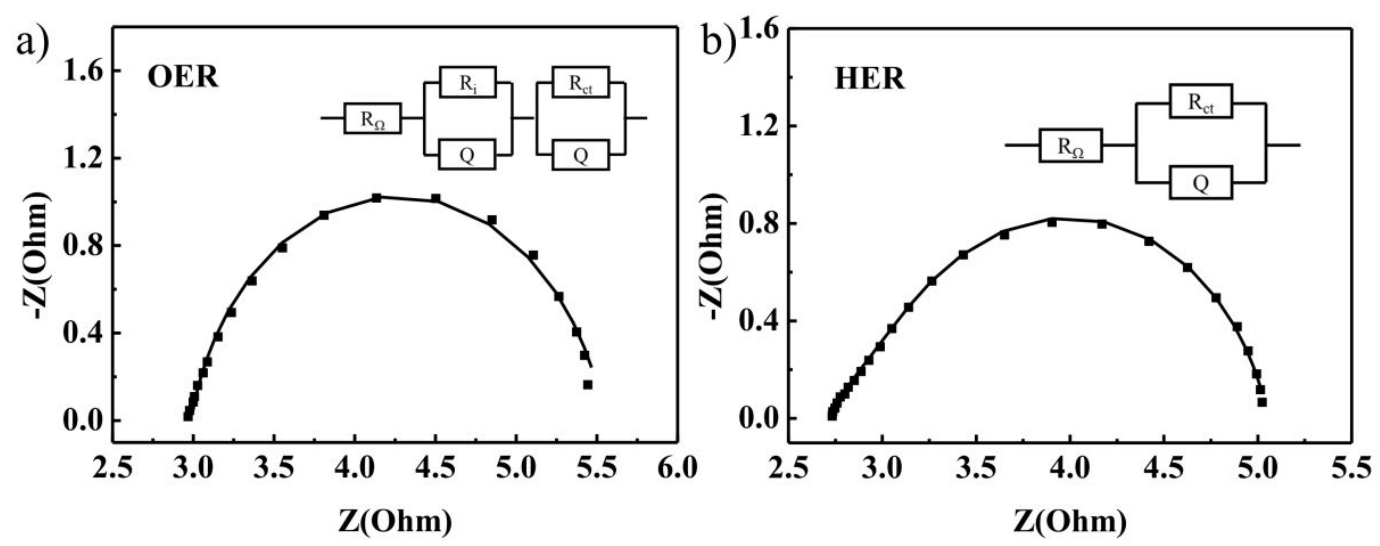

Figure S7. The Nyquist plot of Fe@Ni nanofiber electrode in (a) OER process and (b) HER process. The inset is the equivalent circuit model used for EIS fitting.
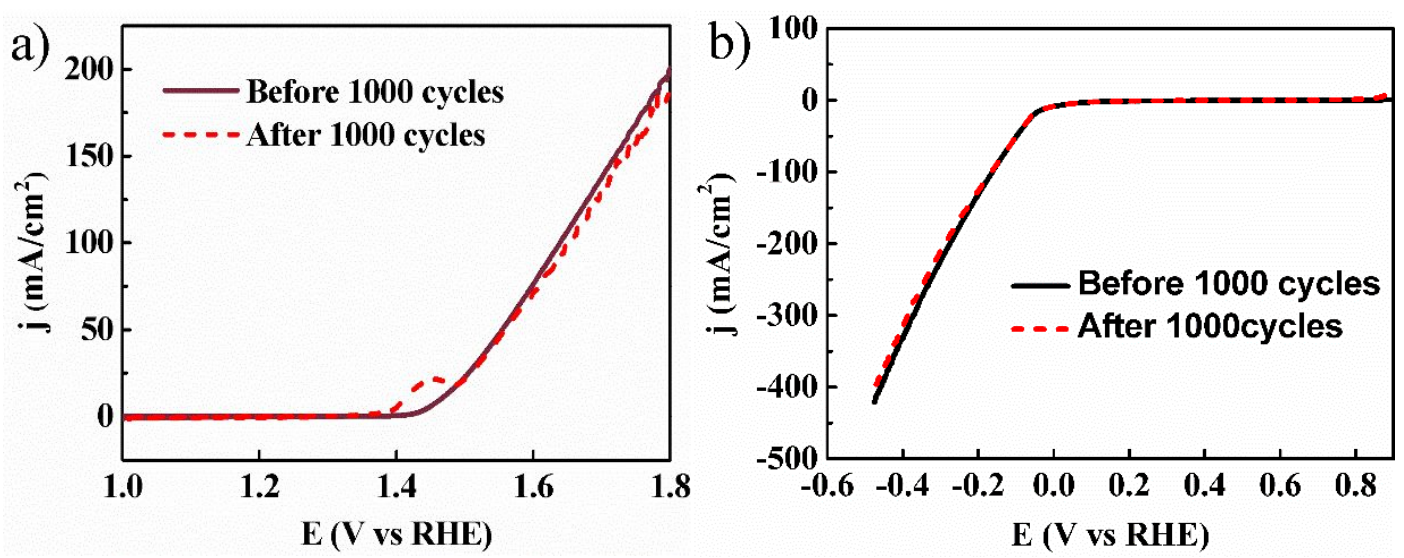

Figure S8. (a) OER and (b) HER durability test for Fe@Ni nanofiber electrode via cyclic voltammetry at a scan rate of $100 \mathrm{mV} \mathrm{s}^{-1}$. 

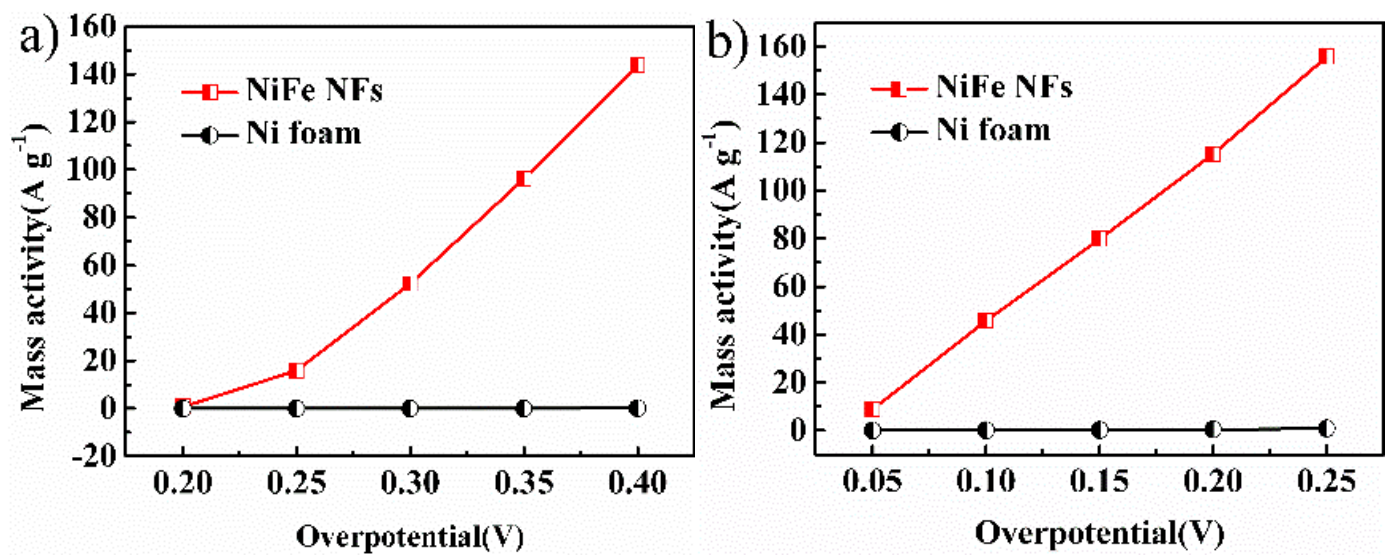

Figure S9. Mass activities of Fe@Ni nanofiber electrode and Fe@Ni foam for(a) OER and (b) HER.
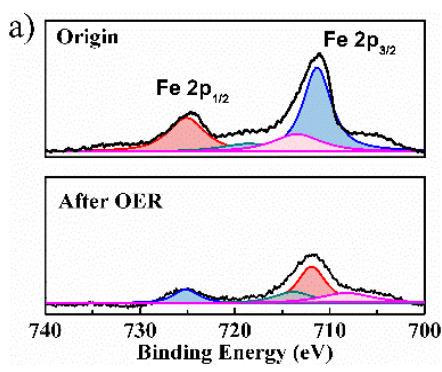
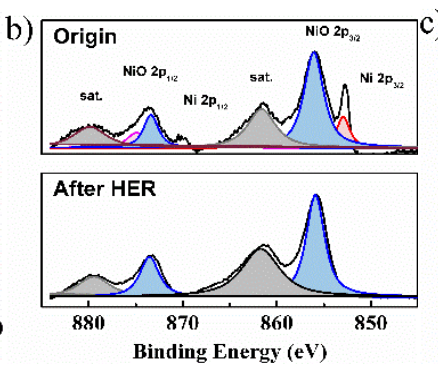
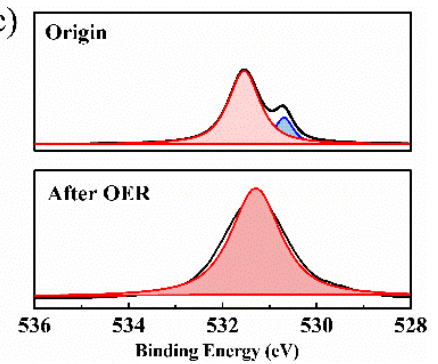

Figure S10. XPS high-resolution scans of Fe@Ni nanofiber electrode before and after

OER reaction: (a) Fe; (b) Ni; (c) O. As can be seen in Figure S10, Ni and Fe are oxidized into higher valence states after the OER process: the binding energy undergoes a shift toward high state in Fe element, and the characteristic peaks of $\mathrm{Ni} 2 \mathrm{p}$ disappeared in $\mathrm{Ni}$ element. The results are in accordance with previous researches on OER reaction, indicating the formation of oxides or hydroxides during and after OER. 

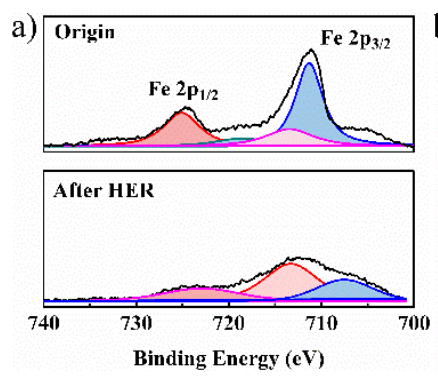
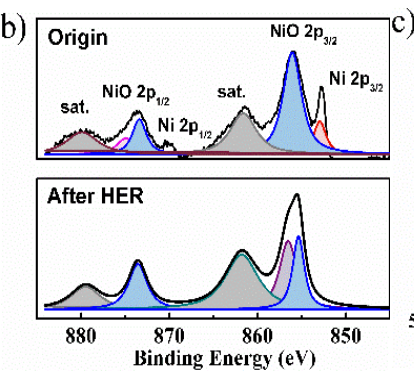
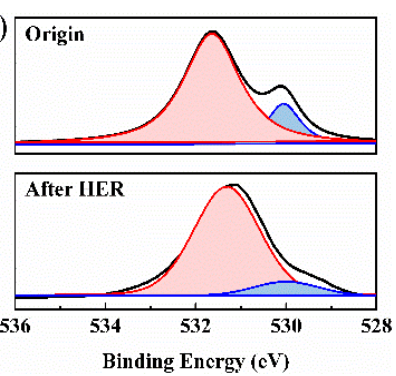

Figure S11. XPS high-resolution scans of Fe@Ni nanofiber electrode before and after HER process: (a) Fe; (b) Ni; (c) O. As shown in above images, metal (oxy)hydroxides can be observed after HER process, indicating the formation of active species. 
Table S1. Elements concentration of Fe@Ni nanofiber electrode by ICP-MS

\begin{tabular}{llll}
\hline & $\mathrm{Ni}$ & $\mathrm{Fe}$ & $\mathrm{Pd}$ \\
\hline Ratio (wt\%) & 99.325 & 0.672 & 0.003 \\
\hline
\end{tabular}

Table S2. The comparison of OER activity of some NiFe-based free-standing electrodes in alkaline electrolyte.

\begin{tabular}{|c|c|c|c|c|c|}
\hline Material & Electrolyte & $\begin{array}{l}\text { Tafel slop } \\
\left(\mathrm{mV} \mathrm{dec}^{-1}\right)\end{array}$ & $\begin{array}{c}\eta \\
(\mathrm{mV})\end{array}$ & $\begin{array}{c}J\left(\mathrm{~mA} \mathrm{~cm}^{-}\right. \\
2)\end{array}$ & Ref. \\
\hline \multirow{2}{*}{ Fe@Ni nanofibers } & \multirow{2}{*}{$1 \mathrm{M} \mathrm{KOH}$} & \multirow{2}{*}{37.5} & 230 & 10 & \multirow{2}{*}{ This work } \\
\hline & & & 268 & 100 & \\
\hline $\begin{array}{c}\text { NiFe LDH on } \\
\text { NiCoP nanowires }\end{array}$ & $1 \mathrm{M} \mathrm{KOH}$ & 48.5 & 220 & 10 & Ref. $^{1}$ \\
\hline NiCoFe MOF & $\begin{array}{l}0.1 \mathrm{M} \\
\mathrm{KOH}\end{array}$ & 49.0 & 320 & 10 & Ref. $^{2}$ \\
\hline \multirow{2}{*}{$\begin{array}{l}\text { NiFe hydroxide } \\
\text { nanoflake arrays }\end{array}$} & \multirow[b]{2}{*}{$1 \mathrm{M} \mathrm{KOH}$} & \multirow[b]{2}{*}{51.5} & 267 & 10 & \multirow[b]{2}{*}{ Ref. $^{3}$} \\
\hline & & & 288 & 100 & \\
\hline $\begin{array}{l}\text { Ni-Fe LDH hollow } \\
\text { nanoprism }\end{array}$ & $1 \mathrm{M} \mathrm{KOH}$ & 49.4 & 280 & 10 & Ref. ${ }^{4}$ \\
\hline \multirow{2}{*}{ NiFeSe cages } & \multirow{2}{*}{$1 \mathrm{M} \mathrm{KOH}$} & \multirow{2}{*}{24.0} & 240 & 10 & \multirow{2}{*}{ Ref. $^{5}$} \\
\hline & & & 270 & 100 & \\
\hline $\mathrm{Ni}_{3} \mathrm{FeN} / \mathrm{Co}, \mathrm{N}-\mathrm{CNF}$ & $\begin{array}{l}0.1 \mathrm{M} \\
\mathrm{KOH}\end{array}$ & 51.0 & 270 & 10 & Ref. $^{6}$ \\
\hline $\mathrm{FeNi}_{4.34} @ \mathrm{FeNi}$ foil & $1 \mathrm{M} \mathrm{KOH}$ & 53.0 & 283 & 10 & Ref. $^{7}$ \\
\hline \multirow{2}{*}{$\mathrm{Ni}_{1.5} \mathrm{Fe}_{0.5} \mathrm{P}$} & \multirow{2}{*}{$1 \mathrm{M} \mathrm{KOH}$} & \multirow{2}{*}{55.0} & 264 & 10 & \multirow{2}{*}{ Ref. $^{8}$} \\
\hline & & & 293 & 100 & \\
\hline \multirow{2}{*}{$\mathrm{NiFe}$ LDH array } & $1 \mathrm{M}$ & \multirow{2}{*}{31.0} & 210 & 10 & \multirow{2}{*}{ Ref. $^{9}$} \\
\hline & $\mathrm{NaOH}$ & & 260 & 100 & \\
\hline \multirow{2}{*}{$\mathrm{Cu} @ \mathrm{NiFe} \mathrm{LDH}$} & \multirow{2}{*}{$1 \mathrm{M} \mathrm{KOH}$} & \multirow{2}{*}{27.8} & 199 & 10 & \multirow{2}{*}{ Ref. ${ }^{10}$} \\
\hline & & & 281 & 100 & \\
\hline
\end{tabular}


Table S3. The comparison of HER activity of some NiFe-based free-standing electrodes in alkaline electrolyte.

\begin{tabular}{|c|c|c|c|c|c|}
\hline Material & Electrolyte & $\begin{array}{l}\text { Tafel slop } \\
\left(\mathrm{mV} \operatorname{dec}^{-1}\right)\end{array}$ & $\begin{array}{c}\eta \\
(\mathrm{mV})\end{array}$ & $\begin{array}{c}J\left(\mathrm{~mA} \mathrm{~cm}^{-}\right. \\
2)\end{array}$ & Ref. \\
\hline \multirow{2}{*}{ Fe@Ni nanofibers } & \multirow{2}{*}{$1 \mathrm{M} \mathrm{KOH}$} & \multirow{2}{*}{53.5} & 55 & & \multirow{2}{*}{ This work } \\
\hline & & & 80.4 & 100 & \\
\hline $\begin{array}{c}\mathrm{NiFe} \text { LDH on } \\
\text { NiCoP nanowires }\end{array}$ & $1 \mathrm{M} \mathrm{KOH}$ & 88.2 & 120 & 10 & Ref. $^{1}$ \\
\hline \multirow{2}{*}{$\mathrm{NiCoFe} \mathrm{MOF}$} & $0.1 \mathrm{M}$ & \multirow{2}{*}{67} & \multirow{2}{*}{270} & \multirow{2}{*}{10} & \multirow{2}{*}{ Ref. $^{2}$} \\
\hline & $\mathrm{KOH}$ & & & & \\
\hline $\mathrm{Ni}_{1.5} \mathrm{Fe}_{0.5} \mathrm{P}$ & $1 \mathrm{M} \mathrm{KOH}$ & 125 & 282 & 10 & Ref. ${ }^{8}$ \\
\hline $\mathrm{Cu} @ \mathrm{NiFe} \mathrm{LDH}$ & $1 \mathrm{M} \mathrm{KOH}$ & 79.7 & 116 & 10 & Ref. 10 \\
\hline $\begin{array}{c}\mathrm{NiCo} 2 \mathrm{~S} 4 @ \mathrm{NiFe} \\
\mathrm{LDH}\end{array}$ & $1 \mathrm{M} \mathrm{KOH}$ & N. A. & 200 & 10 & Ref. 11 \\
\hline \multirow{2}{*}{$\mathrm{Co}_{6.25} \mathrm{Fe}_{18.75} \mathrm{Ni}_{75} \mathrm{O}_{\mathrm{x}}$} & \multirow{2}{*}{$1 \mathrm{M} \mathrm{KOH}$} & \multirow{2}{*}{53.6} & 84. & 10. & \multirow{2}{*}{ Ref. ${ }^{12}$} \\
\hline & & & 192 & 100 & \\
\hline \multirow{2}{*}{$\mathrm{Ni}_{0.7} \mathrm{Fe}_{0.3} \mathrm{~S}_{2}$} & \multirow{2}{*}{$1 \mathrm{M} \mathrm{KOH}$} & \multirow{2}{*}{56} & 155 & 10 & \multirow{2}{*}{ Ref. ${ }^{13}$} \\
\hline & & & $\sim 350$ & 100 & \\
\hline $\begin{array}{c}\mathrm{NiFe}_{2} \mathrm{O}_{4} / \mathrm{NiFe} \\
\mathrm{LDH}\end{array}$ & $1 \mathrm{M} \mathrm{KOH}$ & 30 & 101 & 10 & Ref. ${ }^{14}$ \\
\hline $\mathrm{Ni}_{0.9} \mathrm{Fe}_{0.1} / \mathrm{NC}$ & $1 \mathrm{M} \mathrm{KOH}$ & 100 & 219 & 10 & Ref. $^{15}$ \\
\hline \multirow{2}{*}{$\mathrm{Ni} / \mathrm{NiO}$} & $1 \mathrm{M}$ & \multirow{2}{*}{65} & 46 & 20 & \multirow{2}{*}{ Ref. ${ }^{9}$} \\
\hline & $\mathrm{NaOH}$ & & 137 & 100 & \\
\hline
\end{tabular}


Table S4. The comparison of the two-electrode water-splitting performance of some NiFe-based free-standing electrodes in alkaline electrolyte.

\begin{tabular}{|c|c|c|c|c|c|}
\hline Material & Electrolyte & $\begin{array}{c}\text { Cell } \\
\text { voltage } \\
(\mathrm{V})\end{array}$ & $J\left(\mathrm{~mA} \mathrm{~cm}^{-2}\right)$ & $\begin{array}{c}\text { Catalyst } \\
\text { loading }(\mathrm{mg} \\
\left.\mathrm{cm}^{-2}\right)\end{array}$ & Ref. \\
\hline Fe@Ni nanofibers & $1 \mathrm{M} \mathrm{KOH}$ & 1.53 & 10 & 2.28 & This work \\
\hline $\begin{array}{c}\mathrm{NiFe} \text { LDH on } \\
\text { NiCoP nanowires }\end{array}$ & $1 \mathrm{M} \mathrm{KOH}$ & 1.57 & 10 & 2.0 & Ref. ${ }^{1}$ \\
\hline $\begin{array}{c}\mathrm{NiCo}_{2} \mathrm{~S}_{4} @ \mathrm{NiFe} \\
\mathrm{LDH}\end{array}$ & $1 \mathrm{M} \mathrm{KOH}$ & 1.60 & 20 & -- & Ref. ${ }^{11}$ \\
\hline $\mathrm{Co}_{6.25} \mathrm{Fe}_{18.75} \mathrm{Ni}_{75} \mathrm{O}_{\mathrm{x}}$ & $1 \mathrm{M} \mathrm{KOH}$ & 1.85 & 10 & 4.0 & Ref. $^{12}$ \\
\hline Cu@NiFe LDH & $1 \mathrm{M} \mathrm{KOH}$ & 1.54 & 10 & 2.2 & Ref. ${ }^{10}$ \\
\hline $\mathrm{Ni}_{0.7} \mathrm{Fe}_{0.3} \mathrm{~S}_{2}$ & $1 \mathrm{M} \mathrm{KOH}$ & 1.62 & 10 & 3.0 & Ref. ${ }^{13}$ \\
\hline $\begin{array}{c}\text { Porous Ni-Fe-P@C } \\
\text { NRs }\end{array}$ & $1 \mathrm{M} \mathrm{KOH}$ & 1.52 & 10 & -- & Ref. ${ }^{16}$ \\
\hline $\mathrm{Ni}_{1.5} \mathrm{Fe}_{0.5} \mathrm{P}$ & $1 \mathrm{M} \mathrm{KOH}$ & 1.59 & 10 & 1.38 & Ref. $^{8}$ \\
\hline $\mathrm{NiFe}_{2} \mathrm{O}_{4} / \mathrm{NiFe} \mathrm{LDH}$ & $1 \mathrm{M} \mathrm{KOH}$ & 1.54 & 10 & 2.8 & Ref. ${ }^{14}$ \\
\hline $\begin{array}{c}\mathrm{Ni} / \mathrm{NiO} / / \mathrm{NiFe} \mathrm{LDH} \\
\text { array }\end{array}$ & $\begin{array}{c}1 \mathrm{M} \\
\mathrm{NaOH}\end{array}$ & 1.52 & 20 & $\sim 4.0$ & Ref. ${ }^{9}$ \\
\hline $\mathrm{Ni}_{0.9} \mathrm{Fe}_{0.1} / \mathrm{NC}$ & $1 \mathrm{M} \mathrm{KOH}$ & 1.58 & 10 & 2.0 & Ref. ${ }^{15}$ \\
\hline
\end{tabular}




\section{References :}

1. Zhang, H. J.; Li, X. P.; Hahnel, A.; Naumann, V.; Lin, C.; Azimi, S.; Schweizer,

S. L.; Maijenburg, A. W.; Wehrspohn, R. B., Bifunctional Heterostructure Assembly of NiFe LDH Nanosheets on NiCoP Nanowires for Highly Efficient and Stable Overall Water Splitting. Adv. Funct. Mater. 2018, 28 (14), 1706847.

2. Ahn, W.; Park, M. G.; Lee, D. U.; Seo, M. H.; Jiang, G.; Cano, Z. P.; Hassan, F. M.; Chen, Z., Hollow Multivoid Nanocuboids Derived from Ternary Ni-Co-Fe Prussian Blue Analog for Dual-Electrocatalysis of Oxygen and Hydrogen Evolution Reactions. Adv. Funct. Mater. 2018, 28 (28), 1802129.

3. Liu, J.; Zheng, Y.; Wang, Z.; Lu, Z.; Vasileff, A.; Qiao, S. Z., Free-standing singlecrystalline NiFe-hydroxide nanoflake arrays: a self-activated and robust electrocatalyst for oxygen evolution. Chem. Commun. (Camb) 2018, 54 (5), 463-466.

4. Yu, L.; Yang, J. F.; Guan, B. Y.; Lu, Y.; Lou, X. W. D., Hierarchical Hollow Nanoprisms Based on Ultrathin Ni-Fe Layered Double Hydroxide Nanosheets with Enhanced Electrocatalytic Activity towards Oxygen Evolution. Angew. Chem. Int. Ed. Engl. 2018, 57 (1), 172-176.

5. Nai, J.; Lu, Y.; Yu, L.; Wang, X.; Lou, X. W. D., Formation of Ni-Fe Mixed Diselenide Nanocages as a Superior Oxygen Evolution Electrocatalyst. Adv Mater 2017, 29 (41), 1703870.

6. Wang, Q.; Shang, L.; Shi, R.; Zhang, X.; Waterhouse, G. I. N.; Wu, L.-Z.; Tung, C.-H.; Zhang, T., 3D carbon nanoframe scaffold-immobilized Ni3FeN nanoparticle electrocatalysts for rechargeable zinc-air batteries' cathodes. Nano Energy 2017, 40, 
382-389.

7. Qazi, U. Y.; Yuan, C. Z.; Ullah, N.; Jiang, Y. F.; Imran, M.; Zeb, A.; Zhao, S. J.; Javaid, R.; Xu, A. W., One-Step Growth of Iron-Nickel Bimetallic Nanoparticles on FeNi Alloy Foils: Highly Efficient Advanced Electrodes for the Oxygen Evolution Reaction. ACS Appl. Mater. Interfaces 2017, 9 (34), 28627-28634.

8. Huang, H. W.; Yu, C.; Zhao, C. T.; Han, X. T.; Yang, J.; Liu, Z. B.; Li, S. F.; Zhang, M. D.; Qiu, J. S., Iron-tuned super nickel phosphide microstructures with high activity for electrochemical overall water splitting. Nano Energy 2017, 34, 472-480.

9. Liu, X.; Wang, X.; Yuan, X.; Dong, W.; Huang, F. Q., Rational composition and structure design of the in-situ grown nickel-based electrocatalysts for efficient water electrolysis. J. Mater. Chem. A 2015, 4 (1), 167-172.

10. Yu, L.; Zhou, H.; Sun, J.; Qin, F.; Yu, F.; Bao, J.; Yu, Y.; Chen, S.; Ren, Z., Cu nanowires shelled with NiFe layered double hydroxide nanosheets as bifunctional electrocatalysts for overall water splitting. Energy \& Environmental Science 2017, 10 (8), 1820-1827.

11. Liu, J.; Wang, J.; Zhang, B.; Ruan, Y.; Lv, L.; Ji, X.; Xu, K.; Miao, L.; Jiang, J., Hierarchical NiCo2S4@NiFe LDH Heterostructures Supported on Nickel Foam for Enhanced Overall-Water-Splitting Activity. ACS Appl Mater Interfaces 2017, 9 (18), $15364-15372$.

12. Wu, Z. C.; Wang, X.; Huang, J. S.; Gao, F., A Co-doped Ni-Fe mixed oxide mesoporous nanosheet array with low overpotential and high stability towards overall water splitting. J. Mater. Chem. A 2018, 6 (1), 167-178. 
13. Yu, J.; Cheng, G.; Luo, W., Ternary nickel-iron sulfide microflowers as a robust electrocatalyst for bifunctional water splitting. J. Mater. Chem. A 2017, 5 (30), 1583815844.

14. Wu, Z.; Zou, Z.; Huang, J.; Gao, F., NiFe2O4 Nanoparticles/NiFe Layered DoubleHydroxide Nanosheet Heterostructure Array for Efficient Overall Water Splitting at Large Current Densities. ACS Appl. Mater. Interfaces 2018, 10 (31), 26283-26292.

15. Zhang, X.; Xu, H.; Li, X.; Li, Y.; Yang, T.; Liang, Y., Facile Synthesis of NickelIron/Nanocarbon Hybrids as Advanced Electrocatalysts for Efficient Water Splitting. ACS Catalysis 2015, 6 (2), 580-588.

16. Ahn, S. H.; Manthiram, A., Direct growth of ternary Ni-Fe-P porous nanorods onto nickel foam as a highly active, robust bi-functional electrocatalyst for overall water splitting. J. Mater. Chem. A 2017, 5 (6), 2496-2503. 